\title{
¿Una estrategia pública para la economía andaluza?
}

Manuel Delgado Caibeza*

\section{Introducción}

Existe un acuerdo prácticamente unánime sobre la naturaleza estructural de los problemas que subyacen en la realidad social y económica de Andalucía. Son problemas que hunden sus raices en un largo proceso histórico que ha dado como resultado una determinada manera interna de funcionar a la vez que un modo de articulación con el exterior que define para la economía andaluza unas funciones dentro de la división espacial del trabajo.

Son obstáculos que no solamente revisten gravedad por su agudeza sino también por su carácter crónico y , sobre todo, por su naturaleza, que va más allá de los simples desajustes o arritmias coyunturales, para insertarse en las formas de apropiación, producción, distribución y control de recursos y riqueza. Para superarlos, no bastan alivios sintomáticos. No sería eficaz una terapia superficial; ni siquiera algunas actuaciones parciales y/o aisladas para compensar ciertos desarreglos o desajustes. Este tipo de actuaciones terminan diluyéndose en la propia situación general.

En el caso de la economía y la sociedad andaluza, la naturaleza de los problemas resalta la necesidad de concebir un conjunto de intervenciones coordinadas desde el que se replantee el propio funcionamiento de la totalidad. De modo que, frente a la puesta en marcha de actuaciones singulares, se hace necesario diseñar una estrategia, un planteamiento global, estructurado, que integre los programas, las actuaciones y los proyectos concretos con el fin de alcanzar los objetivos propuestos.

* Quiero agradecer a Carolina Márquez los comentarios hechos a una primera versión de este trabajo.

* Es Catedrático y Director del Departamento de Economía Aplicada II de la Universidad de Sevilla. 
En este sentido, el primer gobierno autonómico andaluz proclama la necesidad que tiene la realidad andaluza de "un conjunto de actuaciones de política económica coherentes entre sí, para lo cual la planificación aparece como el mecanismo adecuado" [Ley del Plan Económico para Andalucía (PEA)1984-86]. Comienza así un proceso de elaboración de Planes para abordar problemas que por su naturaleza "sólo pueden ser afrontados con una perspectiva a largo plazo en la que la planificación se reconoce como el instrumento más eficaz, siendo los distintos Planes secuencias de una misma tarea". [Plan Andaluz de Desarrollo Económico (PADE) 1987-90].

La planificación, por tanto, va a suponer, a través de los diferentes Planes elaborados, tres hasta 1994, la concreción, tanto de la visión que desde el gobierno andaluz se tiene de los problemas que afectan a la economía andaluza como de las soluciones propuestas para abordarlos. En principio, se podría suponer, por tanto, que este es el soporte de una estrategia -término ampliamente utilizado en los Planes-, diseñada para conseguir determinados objetivos.

Pero para que una estrategia pueda ser considerada como tal, se requieren ciertos requisitos mínimos, sin los cuales las actuaciones podrían quedar reducidas a una mera agregación de programas cuyos resultados pueden distar de los objetivos propuestos, o incluso ir en contra de su consecución.

Un primer requisito ha quedado recogido en el párrafo del PADE que acaba de citarse y se refiere a la dimensión temporal de la planificación. Las dificultades y resistencias para vencer los obstáculos al desarrollo exigen un horizonte temporal de largo plazo y a la vez una permanencia en las líneas que definen la propia estrategia. No sería lógico que las metas, los objetivos y los instrumentos estuvieran continuamente en danza, modificándose de un Plan a otro. El carácter secular y la profundidad de los problemas y la envergadura que se requiere para su abordaje justifican una persistencia y cierta estabilidad y firmeza en el mantenimiento del rumbo, es decir, de la estrategia.

Otra condición igualmente razonable viene a ser la de la coherencia a la que se apela en las líneas del PEA que se transcribieron más arriba. Una coherencia que cabe interpretar en un doble sentido. Interna, de modo que dentro de cada Plan, los instrumentos que se proponen mantengan una adecuación con los objetivos planteados, por una parte, y, por otra, que se mantenga la necesaria correspondencia entre el diagnóstico y los instrumentos. El otro tipo de coherencia tiene relación con la dimensión temporal, que ya se ha 
señalado, y hace referencia al contenido de los diferentes Planes entre sí, que debe guardar una concordancia en su contenido, sin que en principio parezcan justificables cambios bruscos ni virajes con respecto al norte señalado, o alteraciones de importancia en los caminos o la dirección a seguir. Como tampoco parecería muy consecuente que los diagnósticos sobre la situación fueran esencialmente distintos en sus planteamientos de unos Planes a otros.

El propósito de este artículo es tratar de contrastar si existe o no una estrategia, en el sentido que acaba de definirse, desde el gobierno autonómico de la Junta de Andalucía, para intentar superar los problemas que la economía andaluza tiene planteados. Este contraste se va a realizar a partir del análisis del contenido de los tres Planes elaborados hasta ahora, en relación con los requisitos o condiciones que acaban de mencionarse.

\section{El Plan Economico para Andalucía. 1984-1986. (PEA).}

Con las reivindicaciones de la autonomía resonando todavía con fuerza y ante la situación de debilidad de la Junta como poder instituido, el Plan Económico, que se va a plantear como un instrumento básico para resolver los graves problemas que la sociedad andaluza tenía pendientes, contribuía, junto con otros elementos, símbolos y gestos utilizados, a justificar y legitimar al propio gobierno del que emanaba.

En este sentido, hay que tener en cuenta también que con el ocaso del poder fundado en la posesión de la tierra, y el correspondiente declinar de la burguesía agraria y ante la falta de un bloque hegemónico, de una fuerza social capaz de elaborar, imponer y mantener su proyecto como el de toda la sociedad andaluza en su conjunto, la Confederación de Empresarios Andaluces, y las organizaciones sindicales "mayoritarias", compartian con el Gobierno andaluz tanto la conveniencia de un discurso "integrador" como el interés por la justificación de su carácter "representativo" como "interlocutores válidos".

La concertación del documento elaborado por la Consejería de Economía con sindicatos y empresarios, contribuye a cumplír este papel de aglutinante del sistema político-económico de Andalucía, reforzando la legitimación de los interlocutores intervinientes en el acuerdo (Barcelay, 1987). 
El Plan andaluz 84-86, en el que, según reza en la presentación, los objetivos y estrategia se sitúan en el contexto de la política económica estatal, " $a$ cuyos objetivos básicos se adaptan todas las políticas económicas incluidas en este documento", comienza con una aproximación a la situación económica y social de Andalucía. Un diagnóstico en el que se describe un conjunto de cifras que se comparan sistemáticamente con los datos correspondientes a la economía española, con la pretensión de establecer los "diferenciales" de desarrollo. Ronda por toda esta primera parte la idea de agravio y de derecho a la igualdad, tan presente en la reivindicación de autogobierno de años todavía recientes.

Así, los rasgos distintivos de la economía andaluza resultan ser "el amplio diferencial a la baja en lo que a la tasas de actividad se refiere"; una mayor tasa de paro o "el peso sustancialmente superior de la agricultura, el débil sector industrial y la mayor importancia del sector servicios". En este mismo capítulo se hace también alusión a otros problemas de alcance estructural, entre los que se citan la débil integración del territorio y del tejido productivo, y la evidencia de recursos productivos no utilizados (p.77).

En consonancia con este tipo de diagnóstico, puramente descriptivo de las diferencias entre indicadores con respecto al modelo de referencia, en el que no se entra en el análisis de las causas, ni del proceso histórico en el que éstas se generan, se plantean como objetivos finales "la reducción del paro y la elevación de la tasa de actividad", "la tasa de paro andaluza debe aproximarse a la correspondiente a la economía nacional"), el incremento del peso relativo de la industria y "un acercamiento de los niveles de equipamiento colectivo a los correspondientes al conjunto nacional", (p.85) y, como paso previo para conseguir estos objetivos finales, como objetivo intermedio "la obtención de un ritmo de crecimiento económico por encima del correspondiente a la economía española"(p.86).

Se trata de conseguir cubrir la distancia en todos los indicadores. Incluso en casos como el de la tasa de actividad, cuyo incremento alejaría las posibilidades de conseguir el objetivo de reducir el paro. Y para ello, a su vez, hay que crecer más que la media.

La visión de los problemas se corresponde aquí, claramente, con el enfoque convencional del desarrollo, que supone la existencia de una línea única en la escalada hacia el progreso, de modo que, de acuerdo con el planteamiento bien conocido de Rostow, las sociedades se sitúan en diferentes etapas 
o peldaños en este ascenso. "Naturalmente", para acortar las diferencias, expresadas en distancias posibles de medir a través de los indicadores correspondientes, basta con crecer más deprisa que los que van por delante.

No voy a insistir aquí, y mucho menos a tratar de fundamentar, algo que en tantos estudios sobre el desarrollo se ha llegado a considerar como obvio, y que para el caso de Andalucía ya está suficientemente argumentado en otros trabajos. Es más, se trata ya de un hecho, porque, por si fuera poco, la experiencia histórica se ha encargado de demostrarlo con rotundidad: el crecimiento sin cambios estructurales profundos no resuelve los problemas de sociedades periféricas como la andaluza. Más aún, termina reproduciendo los mecanismos generadores de la propia situación de partida. Sin entrar en pormenores, los diez años que van desde 1964 a 1973, con el crecimiento económico como protagonista principal de la dinámica económica en Andalucía, cuyo PIB alcanzó una velocidad después no repetida, $16,7 \%$ anual acumulativo) por encima de la media española, pueden ser una buena prueba de ello.

La constatación de la crisis de "las politicas regionales de corte tradicional" lleva a definir un conjunto de actuaciones teniendo en cuenta que: "si de la acción del gobierno central no cabe esperar acciones decisorias para la reducción de los desequilibrios interregionales existentes, el Gobierno autónomo andaluz deberá apoyar su estrategia económica en un esfuerzo movilizador de sus propios recursos productivos", es decir, el llamado "potencial endógeno" (p.89)

Nos encontramos, en principio, ante la elección de un tipo de estrategia para el desarrollo de Andalucía. Profundizemos algo más en el análisis.

En primer lugar, la definición se ha hecho en negativo, es decir, más por dejación de otros, en este caso el Estado, que por convencimiento propio, si bien es cierto que este planteamiento del desarrollo endógeno está muy en la onda con un análisis del subdesarrollo andaluz que encuentra en la dependencia una de las claves para su interpretación y por tanto enfatiza en la necesidad de transformaciones que impliquen una movilización desde dentro. La reivindicación del autogobierno y las demandas sociales y políticas de finales de los 70 y principios de los 80 ponen de manifiesto que este es un enfoque explícita o implícitamente aceptado. En este sentido, puede decirse que hay una coincidencia entre este discurso del Plan y el que se quiere oir. 
Pero esta declaración sobre el tipo de desarrollo por el que se aboga debe tener una coherencia en otros niveles de definición del Plan. Sobre todo, la debe tener a nivel sectorial. Y ahí nos encontramos con que, como estrategia en cuanto a política sectorial, nada más terminar de describir lo que se entiende por desarrollo del potencial endógeno, se propone "un mayor desarrollo de los sectores en los que Andalucia tiene claras ventajas comparativas, como son el subsector agrario, las industrias de transformación agraria, el turismo, y la pesca" (p.90)

El crecimiento, objetivo intermedio del Plan, centrado en los sectores en los que Andalucía tiene ventajas comparativas, viene a profundizar en el modelo que históricamente ha conocido la economía andaluza, y sus resultados son la más clara y evidente expresión de lo que cabría esperar de él. Supone potenciar expresamente los sectores que polarizan la actividad económica en Andalucía, con lo cual se reproducen y se amplían la desarticulación y los desequilibrios sectoriales en un proceso alimentado desde la propia política económica. Hasta tal punto que en el $2^{\circ}$ Plan, el Plan Andaluz de Desarrollo Económico 1987-1990, se llega a decir que la especialización de la economía andaluza en los sectores para los que se tiene ventajas comparativas "es perjudicial para Andalucía" (p.108).

Es posible que esta priorización sectorial que procura más de lo mismo, venga condicionada por un acontecimiento político : el anuncio, al parecer un tanto inesperado, de que el Gobierno Andaluz iba a abordar una de las cuestiones símbólicamente de mayores resonancias en Andalucía: la Reforma Agraria. Una Reforma que luego se definiría en términos que suponían insistir en una modernización que hacía décadas que la agricultura andaluza había emprendido por sí sóla y que iba a terminar quedándose en gran medida en el discurso, es decir, vacía de contenido, si nos atenemos a su incidencia real en la dinámica de la propia agricultura, pero que fué ampliamente utilizada para alimentar la imagen de que por fin se emprendía el camino para superar una cuestión de tan hondas connotaciones en la experiencia colectiva, en la historia de Andalucía.

Pero, volviendo sobre la coherencia en los argumentos contenidos en el Plan, el planteamiento del potencial endógeno es contradictorio e incluso contrario a poner el énfasis en las ventajas comparativas tradicionales. Se trata, en este que entonces resultaba nuevo enfoque, en contra de la teoría neoclásica, de hacer hincapié en ventajas que se relacionan con características 
socioculturales y de comportamiento de la población local asociadas a los procesos de desarrollo.

A pesar de que esta profundización en la especialización productiva tradicional en torno a la agricultura va a terminar prevaleciendo como tendencia y será uno de los rasgos que más claramente definen la evolución de la economía andaluza en las últimas décadas, la opción del desarrollo endógeno tiene sobre el papel su traducción, básicamente, en el programa de ordenación del territorio y el diseño de la red de infraestructuras viarias y en la política de fomento empresarial a través de la creación en 1984 del Instituto de Promoción Industrial (IPIA). Incluso, si nos guiamos por el papel que se asigna al Sector Público, podríamos decir que se adoptan posiciones próximas a lo que desde la teoría de la dependencia se denominó desarrollo autocentrado.

En efecto, en el apartado dedicado a definir "El Sector Empresarial Público", se justifica la necesidad de intervención directa en el terreno productivo por parte del Sector Público, ya que "la iniciativa privada en. Andalucia no ha sido capaz, por muchas razones, de impulsar un desarrollo que acabase con la pobreza y postración andaluzas. La dinámica del subdesarrollo -se continúa diciendo- hace todavia más dificil que pueda asumir ese papel, porque siempre se encontrará en situación desigual, en términos de acceso a financiación, etc... con respecto a colectivos empresariales de otras zonas del Estado o del extranjero. Por estas razones, en una estrategia de desarrollo para Andalucia, el Sector Público tiene que asumir un papel muy importante, tanto para apoyar y dinamizar la iniciativa privada como para llevar a cabo actuaciones de promoción directa"... "la Comunidad Autónoma necesita disponer de un Sector Público con el que actuar de forma beligerante" (p.199) y se comprometía la participación directa del Sector Público en el terreno productivo, dado que "las facultades que el Estatuto de Autonomía confiere a la Junta para crear empresas públicas son muy amplias". Retengamos, por el momento, esta firme intención intervenionista que se manifiesta en el Plan 84-86.

En el Programa de Ordenación del Territorio destacan dos instrumentos diseñados para contrarrestar los desigualdades espaciales en el interior de Andalucía:

10 Después de reiterar ampliamente la gravedad de los desequilibrios territoriales interiores $\mathrm{y}$, reconociéndose que "la Comunidad Autónoma está obligada por el Estatuto de Autonomía (art.12.3.7) y la 
TRABAJO. Revista Andaluza de Relaciones Laborales - n. $\stackrel{2}{2} \cdot$ Diciembre 1996

LOFCA (art.2.2 $2^{\circ}$ a garantizar la solidaridad interterritorial, lo que para las inversiones públicas implica una distribución equitativa de las mismas hasta ahora escasamente practicada" (p.124), se compromete en la Ley del Plan (BOJA 19-6-1984), para conseguir este objetivo, el "Establecimiento de un Fondo de Solidaridad Andaluz para la distribución territorial de las inversiones públicas" (art.46.4). Este Fondo, a cuya descripción se dedican cinco páginas del Plan (126 a 130 ), no se ha vuelto a mencionar desde entonces.

$2^{\mathbf{\alpha}}$ Para poder articular la política de ordenación del territorio se compromete en el PEA (p.288) "la primera medida que se tendrá en cuenta en la política de ordenación territorial: la institucionalización de la comarca como ámbito de acción administrativa y como ámbito, a instancia territorial, de gestión y prestación de servicios públicos"; institucionalización que aún está pendiente.

En el PEA se contemplaba también un Programa de mejora sustancial de las comunicaciones de Andalucía "con el objeto de favorecer una mayor integración de los diferentes territorios de la misma", de modo que en el Avance del Plan General Viario (1984) se diseña una red fuertemente estructurada y centrada en la región, que sustituía la estructura radial por otra en la que se multiplicaban los ejes horizontales y verticales y se conseguía una densa malla interna concebida como instrumento de conexión y ordenación del territorio (Márquez, 1993b)

En materia ferroviaria, el conflicto, analizado en el artículo de Carolina Márquez incluido en este número de la revista Trabajo, entre Renfe, cuyo Plan de reestructuración (1984) suponía una amenaza para toda la red secundaria andaluza, y la Junta de Andalucía, con una posición altamente reivindicativa empeñada en el fortalecimiento de la comunicación intrarregional, refleja también un planteamiento de la política de infraestructuras viarias que se verá modificado sustancialmente a partir de 1987.

Durante este período de vigencia del PEA, se encargaba al Instituto de Promoción Industrial (IPIA) del fomento de la actividad empresarial, que iba a ocuparse de coordinar ciertas medidas encaminadas, a través de los Planes de Actuación, a la dinamización de una zona determinada. Este organismo, que tuvo una existencia corta, con una concepción y una trayectoria muy vinculada a la persona que lo dirigió, actuó expresamente en sectores marginales o residuales, que quedaban fuera de los núcleos polarizadores de la activi- 
dad económica en Andalucía, con intención de atender prioritariamente las necesidades de promoción demandadas desde sectores y actividades tradicionales cuyo proceso de expansión y acumulación pudiera ser controlado desde dentro (Barcelay y O’Kean,1989; Márquez,1993a).

Los Planes de Actuación que se pusieron en marcha fueron los del mármol en Macael, el cuero en Ubrique, el corcho en la Sierra Norte, los azulejos y ladrillos en Bailén, el piñón de Huelva y el Plan de Grazalema. Como puede verse, la incidencia del Instituto fué muy pequeña, apenas perceptible en el conjunto de la economía y la sociedad andaluza. Los escasísimos medios de que dispuso el Instituto en la práctica, -su gasto ejecutado supuso el $0,02 \%$ del presupuesto de los años 84 a 87, como media- pueden también dar una idea de su importancia real.

No obstante, a pesar de esta escasa incidencia, el modo de intervención del IPIA le creó problemas con la Confederación de Empresarios de Andalucía (CECA). Su papel de intermediario entre la Administración y los pequeños empresarios, y su planteamiento de estimular, desde la coordinación, la acción colectiva de éstos, apoyando a empresas que, no siendo precisamente las más eficientes y competitivas de la región, actuaban en el mismo sector o territorio, parece que no fué bien recibido por la CECA, autoconsiderada única representante de todos los empresarios andaluces (Márquez,1993a). De cualquier modo, las molestias no iban a durar mucho. Pronto, en 1986, desaparecería el IPIA y se definiría oficialmente otra manera bien distinta de entender el fomento empresarial.

Con el cambio en la Presidencia de la Junta, y la llegada del nuevo Plan Económico, en 1987, como veremos enseguida, las cosas vienen a ponerse más "en su sitio". Consolidadas las posiciones en el poder, fortalecida y revalidada la legitimación y desactivadas ciertas reividicaciones, se hacía innecesario e incluso podía ser contraproducente, mantener un discurso protagonizado, en el ámbito de lo económico, por el desarrollo endógeno, en un contexto en el que se asistía a una reducción creciente de la endogeneidad decisoria.

Por otra parte, los planteamientos del primer Plan casaban bien con un momento de recesión, de distensión de los lazos que definen la articulación de la economía andaluza con el exterior. Interrumpidas o ralentizadas las correas de trasmisión del crecimiento, se internalizan la responsabilidad y los costes de la atonía, y también de una posible dinamización. 
TRABAJO. Revista Andaluza de Relaciones Laborales - n. $.92 \cdot$ Diciembre 1996

\section{El plan andaluz de desarrollo económico 1987-1990 (PADE).}

Cuando comienzan a recuperarse en el centro las tasas de crecimiento y acumulación, vuelven a funcionar con intensidad en la economía andaluza los mecanismos de articulación con el exterior, dentro de un modelo que se refuerza en las etapas de auge como la que se avecina. Los núcleos polarizadores de la actividad y los espacios más dinámicos, van a volver a atender los requerimientos de la nueva etapa.

Ahora en un contexto en el que, en la economía española, después de los "ajustes" que han tenido lugar en los años de la llamada transición, se profundiza en una modernización definida sobre el eje de una mayor liberalización que permitiera un también más amplio protagonismo del mercado y mayor juego a su papel regulador de la sociedad. Los procesos de desregulación y reestructuración interna, paralelos a la entrada en la entonces CEE, facilitan la inserción en la economía global y la conexión con los ámbitos donde tienen lugar los principales procesos de crecimiento y acumulación.

En este panorama se desenvuelve la elaboración del segundo Plan (PADE 87-90) a cuyo contenido nos vamos a referir en este apartado.

En la introducción de este PADE 87-90 se hace hincapié en la necesidad de que los diferentes Planes formen parte de una misma estrategia, con una perspectiva temporal mantenida que permita abordar los problemas estructurales de la economía andaluza. Esta imprescindible continuidad parece que exigiría, entre otras cosas, comenzar cada Plan con un balance sobre los resultados conseguidos en el anterior. Así se reconoce en el PADE 87-90, donde puede leerse que "la elaboración de un Plan ha de partir del juicio critico que merezca la ejecución del anterior" (p.288).

Después de este reconocimiento expreso, puede sorprender que el primer capítulo del PADE verse sobre el "Análisis general y comparado de la economía andaluza", sin ningún juicio crítico sobre la ejecución del Plan anterior. Aunque, de este modo, se evita entrar en la evaluación de hasta qué punto se cumplieron los objetivos y se instrumentaron los medios para conseguirlos. A lo largo del PADE no se hace referencia al contenido del PEA, ni, por tanto, al grado de cumplimiento de los compromisos adquiridos en él 
Aunque en el nuevo Plan se afirma que la presentación que se hace de la situación de la economía andaluza no es sustancialmente distinta de la que se hizo en el PEA 84-86, pueden encontrarse aquí elementos que no figuraban en el Plan anterior y que modifican de manera importante el diagnóstico, cambiando la estructura lógica de la que deben deducirse las propuestas de actuaciones.

En este sentido, dentro del análisis de los principales problemas, se dedica un apartado a la débil estructura empresarial, identificándose esta debilidad como un factor clave, condicionante a su vez de la ausencia de un tejido industrial denso, fuerte e integrado que pueda generar valor añadido -riqueza- y dar lugar a un cículo virtuoso semejante al que tiene lugar en las economías desarrolladas.

En la misma línea, a lo largo del PADE se señala insistentemente como factor clave entre los obstáculos al desarrollo la falta de reinserción en el circuito económico de Andalucía de una parte del excedente que en ella se genera, obstáculo que provoca, entre otras cosas, que los efectos difusores del crecimiento no tengan lugar aquí.

Por eso, cuando se hace referencia a la necesidad de conseguir como objetivo el incremento de la renta y una mayor integración del sistema productivo, ahora se señala que "no basta con aumentar el valor añadido generado por la economía andaluza: es necesario que ese valor añadido se retenga en Andalucia" (p.104). Este planteamiento lleva a la desaparición del crecimiento económico como objetivo explícito del PADE.

"Es bien conocido -se continúa diciendo (p.104)- que importantes volúmenes de renta no llegan a producirse en nuestra Comunidad Autónoma por la escasa integración, tanto sectorial como espacial, de su sistema productivo, con la consiguiente falta de efectos multiplicadores sobre la renta y el empleo. También algunas deficiencias de nuestra estructura económica, tales como el control empresarial exterior de una parte de la misma y la ineficiencia provocada por un fuerte minifundismo empresarial son importantes limitaciones a la retención de la renta generada".

Pero, por una parte, el fuerte minifundismo, a su vez, está relacionado, según el PADE, con "la existencia de lo que podríamos denominar ‘bloqueo empresarial producido por las dificultades a que se enfrenta el empresariado autóctono no sólo para introducirse en el mercado, sino también para competir 
con el de otras zonas del Estado y del extranjero" (p.34) y por otra, "la escasa articulación de la economía andaluza viene determinada en gran parte por el control empresarial exterior" (p.29). Este diagnóstico, por tanto, entraría en clara contradicción con cualquier propuesta de desarrollo exógeno basada en la atracción de grandes empresas del exterior.

Del análisis que se hace a lo largo del PADE de la situación económica de Andalucía puede deducirse claramente que en la identificación de las causas de esta situación, ocupan un lugar central un conjunto de factores histórico-estructurales que han definido un modo de articularse con el exterior que los mecanismos del mercado han ido reforzando de manera progresiva. De modo que la superación de este modelo de extraversión se contempla en el Plan como imprescindible para la consecución de los otros dos objetivos (creación de empleo y mejora de la calidad de vida) y por tanto se sobreentiende que debería convertirse en el nudo gordiano de una posible estrategia de desarrollo para Andalucía.

Pues bien, lo lógico desde el análisis realizado en el PADE, con independencia ahora de que se esté o no de acuerdo con él, parecería diseñar un conjunto de actuaciones encaminadas a potenciar un modelo de acumulación cualitativamente distinto, un modelo que permita integrar en el circuito económico interno un conjunto de elementos para favorecer la autoalimentación del mismo,al propio tiempo que se internaliza el control de la economía.

Sin embargo, de repente nos encontramos con que se le da al problema otra solución bien distinta a la que se deriva del propio análisis de los problemas, dado que "estos problemas son dificilmente abordables en una economía de mercado en la que además soplan vientos de "desregulación". En este contexto, y puesto que en Andalucia es aún tarea prioritaria sentar las bases fisicas del desarrollo, se considera necesario realizar un notable esfuerzo en la creación de infraestructura durante los próximos años. Este es, por lo demás, el instrumento más potente y más conveniente en este momento que puede utilizar el ejecutivo Andaluz para actuar sobre todos estos problemas" (p.104).

Utilizar como instrumento más potente para superar los obstáculos que la economía andaluza tenía planteados, la inversión en infraestructura, sólo hubiera podido justificarse si la causa de ese modelo de acumulación, localizado en el PADE como núcleo principal de los problemas, estuviera en la falta de infraestructura; si el elemento que encontráramos en la raiz, en el origen 
de la situación, fuera la falta de vertebración provocada por la escasez de vías, de articulación.

Pero en el diagnóstico del PADE se dice justamente lo contrario. La razón de la desarticulación hacia adentro es la forma de inserción con y en el exterior, de tal manera que, de este particular modo de estructurarse la economía andaluza hacia fuera ha resultado una red de comunicaciones "concebida principalmente para servir de enlace con el exterior" (PADE p.35).

Podríamos plantear aquí a propósito de la solución propuesta, sin un proceso paralelo de transformación en la forma en que se estructura, lo cual significa cambios profundos en la forma en que se produce y se distribuye la riqueza, a quién beneficia mejorar las condiciones de acceso ¿no será a quien en mejor disposición esté para aprovecharlas, que no es precisamente el raquítico empresariado andaluz? Porque por esta vía podríamos llegar a la conclusión de que lo que en otro contexto podría ser un elemento liberador y potenciador de una economía, se puede convertir en economías como la andaluza, si a la vez continúa el proceso de deterioro del tejido empresarial, en un elemento alimentador de dicho proceso. Tal vez sean estas razones las que lleven al elaborador del PADE a decir que : "la oferta de infraestructura adecuada es condición necesaria, pero no suficiente para el desarrollo regional (hay ejemplos ya tópicos sobre los efectos negativos de tales politicas)" (p.104).

Más aún, nos podríamos preguntar qué ocurriría si, encima, la infraestructura que se dota insistiera en un diseño que se ha considerado "inadecuado" desde el propio Plan. Porque, tanto en este Plan como en el PEA 84-87 se llega a la conclusión de que insistir en el modelo viario anterior, concebido para atender necesidades ajenas y que descuida la vertebración interna para facilitar la conexión con el exterior significa profundizar la desarticulación y en definitiva reforzar la dependencia. De acuerdo con este planteamiento, según los Planes de infraestructura regionales elaborados antes de 1987 desde la Junta de Andalucía, la red viaria andaluza debía modificar su norte, cambiar su epicentro, atendiendo prioritariamente a las necesidades que demandaba la articulación y la vertebración interior.

La segunda mitad de la década de los 80 supuso en la práctica, un cambio de rumbo con respecto a estos planteamientos. El Programa de carreteras de la Junta de Andalucía, abandonando las prioridades establecidas en el Avance del Plan Viario de 1984, desde 1987 se centró, básicamente, en un sólo 
proyecto: la Autovía del 92, que absorbió aproximadamente el 50\% del total de recursos entre 1987 y 1990.

Se trazaba un pasillo o corredor, la A-92, perteneciente a un modelo de ordenación del territorio distinto al concebido en la etapa anterior, que respondía más a un esquema territorial estatal y europeo. Un complemento de las de Madrid a Sevilla, A-92 a Málaga, o Autovía del Mediterráneo hasta Almería, itinerarios fundamentales para conectar los principales polos de crecimiento y acumulación regional con el exterior (Márquez,1993b).

Se profundizaba así un modelo que en los dos Planes se había identificado como reproductor y alimentador de la polarización, la desarticulación y los desequilibrios territoriales, desde una "centralidad" de la infraestrura, que, cambiando la orientación que se consideraba adecuada, se convierte, además, en foco de atención prioritario de la Junta. Resulta difícil de entender la falta de correspondencia entre diagnóstico e instrumentos en el PADE, la inadecuación entre la naturaleza de la enfermedad detectada y la terapia que se recomienda para combatirla, a no ser que esta "centralidad" de la infraestuctura estuviera ya "comprometida", de antemano y por encima de la elaboración del Plan.

En este momento, en el que la construcción del Mercado Único y la consiguiente eliminación de barreras para facilitar su creación están en el centro de preocupación de las políticas de la CEE, las infraestructuras viarias constituyen el núcleo prioritario de las estrategias de desarrollo regional que emanan de Bruselas, como lo es para el gobierno español, que señala como primera línea de actuación dentro de la estrategia para Andalucía "el papel de las infraestructuras técnicas y equipamientos de base que faciliten las comunicaciones con el centro y norte de la península, pero también que conecten la región de Andalucía con el eje Mediterráneo a través de Murcia y permitan la articulación de la región andaluza en el sentido Este-Oeste". (Ministerio de Economía y Hacienda, 1989:47)

Esta estrategia sintoniza bien con el reparto de papeles que se hace en el PADE 87-90 y que también supone un cambio importante en relación con el definido en el Plan anterior. El Sector Público se retira de la arena en la que antes se pensaba, de un modo firme y rotundo, que necesariamente debía intervenir directamente para suplir las deficiencias y debilidades de la iniciativa privada, y pasa a desempeñar una función de "apoyo y fomento", más adelante veremos a quién. En cambio, ese sector privado, sobre el que, no 
sólo se comparte con el PEA 84-86 la preocupación por su debilidad, sino que ésta se asocia a las dificultades que, como resultado de un largo proceso histórico, tiene para competir frente a otros, ahora tendrá que asumir el protagonismo exclusivo en la lidia.

De modo que, a continuación de varios párrafos en los que se insiste en las importantes limitaciones del empresariado andaluz y en los efectos perniciosos del control exterior, se concluye que "en definitiva será el sector privado el que habrá de jugar un papel decisivo. El empresariado andaluz va a tener una oportunidad inmejorable de mostrar su capacidad real de actuación” (p.106).

Con independencia de la experiencia histórica, parece poco congruente que, después de señalar al control empresarial externo y al bloqueo empresarial en que se encuentra la pequeña y mediana empresa andaluza, por su situación de desventaja en los mercados, como factores claves para explicar la situación actual, se proponga como remedio dejar a la interperie al débil tejido empresarial andaluz como algo "estimulante". En el marco de la globalización y el Mercado Único, extremadas las condiciones de la competencia, con grandes conglomerados empresariales modulando y controlando en gran medida los procesos de crecimiento y acumulación, estaríamos en el "más difícil todavía".

La política de promoción industrial se distribuye entre el Instituto de Fomento de Andalucía (IFA), la Dirección General de Industria y la Dirección General de Cooperación Económica. Centrando la atención en los Incentivos Regionales, "principal instrumento legal que canaliza las ayudas a la inversión empresarial" (Consejería de Economía y Hacienda, 1990), su distribución para el período 1988-90 muestra una fuerte desigualdad, de tal modo que las 30 mayores subvenciones, de las más de 800 que se conceden, suponen el $55 \%$ del total subvencionado. Este dato "relativiza el discurso sobre el apoyo de los incentivos a las Pequeñas y Medianas Empresas" (Marchena y Gómez,1991).

En este segundo Plan puede decirse que estamos ante un Plan mixto o, como veremos, de transición, que, después de un diagnóstico, diferente al que se presentaba en el PEA 84-87, con el que sería más congruente una estrategia de desarrollo endógeno próxima a la que se proponía en el Plan anterior, se apunta en gran medida hacia el desarrollo exógeno, modelo que va a cobrar mayor importancia en el Plan siguiente. 


\section{El plan andadaluz de desarrollo económico 1991-1994.}

En el PADE 91-94 vuelve a echarse en falta un balance sobre los resultados de los planes anteriores; no hay ninguna evaluación de la medida en que se utilizaron los instrumentos y su contribución a la consecución de los objetivos fijados, o hasta dónde llegó el grado de realización de lo comprometido. Hay, eso sí, un capítulo sobre la planificación económica en Andalucía para justificar la necesidad de utilizar la planificación como instrumento, sin una base argumental fundada en un análisis de la contribución de los planes precedentes a la superación de los problemas estructurales que la economía andaluza viene padeciendo.

La "modernización", como referente, ocupa en este Plan un primer plano, situándose a lo largo del mismo como uno de los aspectos centrales del discurso. Esta es una invocación que expresa, ahora en otros términos, una aspiración paralela a la que se traducía en el PADE 84-87 en la pretensión de acortar los llamados "diferenciales" de desarrollo. Se trata, en versión más moderna, de trasplantar a una realidad como la andaluza los elementos, rasgos o aspectos que caracterizan a las economías desarrolladas, reproduciendo en ella ciertas formas tecnológicas y de organización que se están imponiendo en las sociedades que son percibidas como modelo de modernidad. De modo que este es un ímpetu modernizador que tiene como referente básico el nuevo modelo de desarrollo capitalista que se ha definido en el centro del sistema como respuesta a la crisis del fordismo.

Esta visión, desde la que se hace uso con frecuencia de términos como "menor desarrollo relativo" para aludir a la situación de sociedades y economías periféricas, presupone que el "atraso" en esa senda única hacia el desarrollo es consecuecia de la falta en dichas sociedes de una serie de ingredientes o factores, ante cuya ausencia es necesario "establecer las condiciones" que procuren su llegada. En lo fundamental, por tanto, se trata de salvar una distancia, y en este sentido, el crecimiento, abandonado como objetivo en el Plan anterior, vuelve a ser actor principal en el escenario que se define en éste.

Para conseguir la modernización en el sistema productivo, "dirigida a que la economía y la sociedad andaluza se adapten a las actuales exigencias de competitividad que requiere la creciente apertura de los mercados"(p.19), el Sector Público andaluz asumirá las funciones "tal como se entiende hoy el 
papel que deben representar los poderes regionales en el desarrollo económi$c o$ " (p.27),o, dicho de otra forma, tal como ha sido definido en los espacios que se tienen como modelo de referencia. En el ámbito económico, el sector público andaluz asumirá el papel de prestador de servicios como infraestructuras, formación de recursos humanos, apoyo a las nuevas tecnologías, y otros incentivos, encaminados a "impulsar las potencialidades de los factores de competitividad regional" (p.26). En el propio Plan se reconocen las limitaciones de este tipo de actuaciones, en un cotexto en el que "se estrechan los márgenes de maniobra en materia de politica económica"(p.18).

Recordemos que, en el PEA 84-87 se afirmaba rotundamente que la iniciativa privada en Andalucia no sería capaz de superar una situación que se calificaba de subdesarrollo (aunque, según el nuevo PADE 91-94, esta es una expresión que se había venido utilizando "quizá exageradamente" p.67). En el párrafo del PEA 84-87 citado en el apartado 3, se llegaba incluso a expresar la idea de que esa situación, dejada al libre juego de las fuerzas del mercado, se autoalimentaba.

Desde esta lógica, en un contexto de apertura, eliminación de barreras y un mayor protagonismo del mercado, en el que los más débiles quedan aún más desprotegidos, el nuevo papel del Sector Público, que no sólo no contraria las leyes del mercado sino que las refuerza facilitando las condiciones a quienes estén en mejores condiciones de aprovecharlas, mediante el apoyo y sostén a situaciones mercantiles eficaces, supone de hecho la renuncia a las transformaciones estructurales que se habían venido considerando como necesarias, tal vez en la confianza de que éstas puedan llegar traidas directamente por la mano invisible.

En este sentido puede leerse en el Plan que "la mayor liberación, la eliminación de obstáculos a la competencia interna y la creciente competitividad entre los diversos espacios económicos para obtener ganancias potenciales puede suponer para Andalucia una mayor posibilidad para desarrollar sus ventajas potenciales con respecto a otros espacios" (p.95)

En el diagnóstico sobre la economía andaluza que se hace en el capítulo 3 , no es extraño que, tratándose, como se vió, de un problema de acortar distancias, se vuelva ahora a otorgar un especial protagonismo al crecimiento para valorar la dinámica económica en el período 85-90, de modo que la mejora en los resultados coyunturales de algunos indicadores que luego se han derrumbado de nuevo en la etapa de vigencia de este Plan, sirve para cons- 
truir una imagen positiva y optimista sobre la evolución de los problemas que la economía andaluza tiene planteados.

Desde la ignorancia del análisis de la situación que se hizo en el PADE 87-90, y sin que ésta sea sustancialmente distinta, ahora se elabora un diagnóstico de otro tenor, por tercera vez distinto, en este tercer Plan. Desaparecen de la escena los que en el anterior Plan aparecían como los artífices de la situación económica de Andalucía, es decir la extraversión, el control empresarial exterior o el bloqueo empresarial resultante de las condiciones en que históricamente se había desenvuelto la competencia para el tejido autóctono.

Por otra parte, en relación con la estructura productiva se insiste en la descripción de características ya conocidas, orillándose cuestiones cuyo conocimiento debía ser básico, dada la preocupación central por mejorar la competitividad, como ¿cuáles son las funciones que nuestra estructura productiva cumple en ese nuevo orden económico que se está generando? ¿cómo han evolucionado estas funciones en los últimos años? ¿cuáles son el estado y las tendencias de la capacidad competitiva en las distintas actividades y sectores? ¿está la estructura productiva andaluza más fuerte y en mejores condiciones para competir en condiciones de igualdad que en períodos anteriores ? Y sobre todo ¿cuáles son las razones que hay detrás de las respuestas que puedan tener estas interrogantes?

Porque mientras más se aleje el diagnóstico de un análisis causal que permita identificar las raices de los problemas, mayores son las posibilidades de dejarse arrastrar por voluntarismos sin fundamento que lleven a hacer propuestas inviables. En este sentido, se derraman por el Plan posibilidades, espectativas, oportunidades, potencialidades, impulsos, tal vez bajo los efectos de la euforia de la reciente etapa alcista por la que acaba de pasar la economía andaluza que lleva a suponer que se están produciendo algunos cambios como "la aparición -en el sector industrial- de ciertos complejos emergentes para los que Andalucía representa algunas ventajas comparativas" (p.95) sin que en el diagnóstico se haya desvelado cuál es la naturaleza del crecimiento y sobre todo hasta qué punto las características y el modelo al que se ajustaba era semejante al que se había conocido en otros períodos.

Períodos recientes en los que la economía andaluza había crecido, en una etapa doble en duración, por encima de lo que lo ha hecho en la segunda mitad de los 80 , siendo el sector industrial también el que, como en esta oca- 
sión, se convirtió en el "protagonista más significativo del crecimiento de la economia andaluza" (p.83), con una intensidad que estuvo entonces incluso por encima de la que ahora se había conocido. Pero la historia se repetía también en cuanto a la naturaleza del crecimiento y al modelo que lo regía, como se puede deducir de algunos de los Informes de Coyuntura publicados en aquellos años por la Junta de Andalucía (especialmente los números 2 y 3 de 1989 ) y en trabajos posteriores (Morillas Raya, 1995; Delgado Cabeza, 1995).

En cuanto a los objetivos, los que se consideran en el PADE 91-94 no coinciden con los planteados en Planes anteriores. Las diferencias más importantes serían, por una parte la no consideración ni de la creación de empleo ni de la reducción del paro entre las metas y/o los objetivos. La disminución del paro, que fué primer objetivo en el PEA 1984-87 y la creación de empleo, que es una forma menos comprometida en su planteamiento, expresado así como primer objetivo del PADE 87-90, no aparece ahora en el PADE 91-94 ni entre las dos metas ni entre los 15 objetivos que figuran en el Plan. Sorprende esta ausencia en un contexto en el que el paro no había dejado de crecer.

Ni siquiera parece justificable la exclusión del empleo entre los objetivos si lo de que se esperaba era que el crecimiento pudiera solucionar el que se viene considerando, con un amplio consenso al respecto, como el principal problema de la sociedad y la economía andaluza. En primer lugar porque en otras épocas de fuerte crecimiento no se había resuelto de por sí, el problema, sino todo lo contrario.Y por otra parte, porque el crecimiento de la productividad en la economía andaluza viene produciendo una disminución en la ya escasa capacidad de ésta para generar empleo, acentuada en la década de los 80 , que lleva a que cada vez se pueda confiar menos en el crecimiento como mecanismo generador de empleo.A esta disminución de la capacidad para generar empleo de la economía andaluza tampoco se hizo referencia en el diagnóstico del PADE 91-94.

También hay que señalar como diferencia en relación con lo anterior, la reaparición en el PADE 91-94 del crecimiento como primera meta a alcanzar, aunque esta vez se acompaña de dos adjetivos: sostenible y equilibrado. En la justificación del crecimiento, que había desaparecido como objetivo del Plan anterior, se afirma que "el acercamiento a los niveles de desarrollo logrados en nuestros paises de referencia pasa por alcanzar unas tasas de crecimeinto económico relativamente elevadas, manteniendo en lo posible el diferencial de crecimiento habido en años anteriores con respecto a las áreas de nuestro entorno" (p.101) 
No mucho tiempo despuès, la experiencia histórica y los trabajos sobre lo sucedido en la década vendrían a mostrarnos el balance de esa etapa de fuerte crecimiento económico. Pero más pronto aún, poco tiempo después de la entrada en vigor de este PADE 91-94, quedaría claro el grado de control que la Junta de Andalucía tiene sobre el crecimiento que se fijaba como primera meta. Se entraba en una etapa profundamente depresiva en la que, entre otras consecuencias, se destruía empleo a una media de casi cuarenta mil al año. Esta capacidad para "pilotar" la economía en cuanto a cantidad de crecimiento nos puede orientar e ilustrar también sobre las posibilidades de definir su calidad y decidir sus atributos.

No obstante, y a pesar de que la escasez de medios y la disminución de la capacidad de maniobra y de incidencia en la realidad a la que se hacía referencia en los primeros capítulos del Plan, parecían aconsejar ser muy selectivos en la elección de los fines, siendo, por esencia, la planificación un proceso asociado a la priorización y la selección como elementos definitorios, en el PADE 91-94 se fija una más amplia gama de objetivos que la que se elige en Planes anteriores. Esta abundancia en los fines, que en principio proporciona una mayor consistencia y solidez al Plan sobre el papel, diluiría, en buena lógica, las repercusiones, que ya de por sí se han supuesto limitadas, de los medios que se proponen.

Cambios en los objetivos y también en las propuestas. En este sentido, en relación con la política de sectores, a la que se dedica el capítulo 5, el Plan elaborado para el período 91-94 "apuesta por situar a Andalucía en la futura división internacional del trabajo como un espacio económico capaz de producir bienes manufacturados competitivos, y se opta por una economía industrial" (p.128), tratándose, de "perseguir una diversificación basada en una estrategia de desarrollo exógeno" concentrada en la atracción de empresas y, a la vez, como actuación complementaria, "la preservación de las ventajas competitivas de los sectores en los que el grado de especialización de Andalucía es mayor".

Según se dice en el Plan, la diversificación de la industria necesita la identificación de las agrupaciones en las que ésta "es realmente posible". Y utilizando como criterio el de las expectativas de evolución de los mercados, se supone que la diversificación será "realmente posible" si se opta por las actividades cuyos mercados se encuentran en expansión. Estas serán las actividades "en las que Andalucía tiene mayores oportunidades de mejorar posiciones relativas dentro del contexto nacional, y, con esto, lograr mayores nive- 
les relativos de industrialización". Aunque "son precisamennte aquellas en las que Andalucia cuenta con menores ventajas competitivas". Por esta razón, "la estrategia se tiene que concentrar básicamente en la atracción de empresas que ya cuentan con estas ventajas y tienen superadas $e$ imponen fuertes barreras de entrada"(p.129).

Se presume que, en principio, desde esta opción, elegida a partir de un razonamiento en abstracto, que podría haberse hecho en cualquier otro espacio, y, compitiendo con otras regiones (p.133), puede conseguirse que sea aquí donde se localice un conjunto de grandes empresas alrededor de las cuales se nuclee un denso y dinámico tejido empresarial.

Sin entrar en la distancia entre este diseño y la realidad industrial andaluza en los cuatro años de vigencia del Plan, 1991-94, el caso es que el desarrollo exógeno adquiere, explícitamente, en el PADE 91-94, un papel preponderante, y en este sentido nos encontramos en las antípodas de los Planes anteriores. No sólo del PEA 84-86, en el que, como se vió, se subrayaba la necesidad de una estrategia de desarrollo endógeno, sino también del PADE 87-9O, que señalaba el control exterior como un obstáculo clave para explicar la situación actual de la economía andaluza.

\section{Consideraciones finales.}

Como muestran los trabajos más recientes al respecto, la realidad económica y social de Andalucía, ha discurrido por derroteros muy distintos a los pretendidos en los Planes. De modo que, objetivos que se han propuesto en los mismos con insistencia han quedado lejos de ser alcanzados. Por citar algún caso concreto, la distancia entre el mayor equilibrio territorial en el interior de Andalucía que se proponían conseguir los Planes y lo sucedido en el medio rural andaluz puede resultar ilustrativo como ejemplo. En este sentido, en uno de los Planes se llegaba a decir: "es posible orientar la ubicación de algunas actividades hacia las zonas de baja actividad industrial".

Sin embargo, en el caso de la actividad industrial más importante, con mucho, en Andalucía, la industria agroalimentaria, la distribución espacial de las inversiones en el período 1984-1994 nos muestra una fuerte concentración en muy pocos municipios - 18 de los 769 existentes absorben más del 
TRABAJO. Revista Andaluza de Relaciones Laborales - n. ${ }^{2} 2 \cdot$ Diciembre 1996

$60 \%$, mientras que la gran manayoría del territorio andaluz ha permanecido al margen de los procesos de crecimiento y acumulación. (Degado Cabeza y Román del Río,1995). Estos son los resultados finales de una dinámica económica en la que el mercado, desde su acentuado protagonismo, impone los patrones de comportamiento y termina por encargarse de la ordenación del territorio en Andalucía. En éste, como en otros casos, el recorrido ha sido en dirección contraria.

En el camino que se ha seguido hasta aquí, sin entrar en otras custiones que podrían desviar nuestra atención del objetivo propuesto (correspondencia entre el contenido de los Planes y los programas de inversión realizados, o la selección de inversiones), se ha tenido ocasión de constatar la abundacia de incoherencias y contradicciones, en medio de repentinos cambios y bruscos virajes a la hora de definir una política económica en la que aparecen instrumentos que en lugar de estar en corcondancia con unos determinados objetivos, parecen decididos con independencia del análisis que se hace de la realidad económica de Andalucía y de los fines que se dice perseguir.

Todo esto cuando, como se ha señalado en los propios Planes, ante problemas de naturaleza estructural, se supone mayor la necesidad de responder con un diseño global a largo plazo. Una estrategia que debe estar íntimamente conectada con las metas que se proponen, con los plazos para alcanzarla y con los costes que exige, $\mathrm{y}$, por tanto, debe decidirse al más alto nivel, y cuya responsabilidad no debe delegarse en consultores externos. En este sentido, los diferentes Planes traducen también distintas ópticas, diversos modos de interpretar los problemas y propuestas dispares para abordarlos. Se trasluce así, en su análisis, un alto grado de desorientación en las alturas, una falta de pautas de navegación en correspondencia con los problemas que la región tiene planteados. En definitiva, la ausencia de un rumbo, un norte que apunte hacia la superación de los problemas existentes.

La pregunta que cabe hacerse a continuación es ¿a qué intereses sirve entonces la política económica que asume el gobierno andaluz? Porque, aunque en principio pudiera dar la impresión de que se va "a la deriva", esta aparente falta de estrategia se convierte de hecho en la estrategia más contraria a los intereses del desarrollo de Andalucía.

En este punto habría que empezar por plantearse algunas cuestiones sobre el propio papel de la planificación en economías como la andaluza, como 
¿hasta dónde cabe esperar que desde el Sector Público se modifique una dinámica económica que es el resultado y a su vez está gobernada, cada vez en mayor medida, por el protagonismo del capital privado y el libre juego de las fuerzas del mercado? ¿se puede diseñar una operación de "ingeniería social" la planificación es, en esencia, eso- que contrarie las tendencias predominantes, en medio de unas condiciones en las que, al mismo tiempo se busca consolidar e incrementar la preponderancia del mercado en la regulación del funcionamiento general del sistema? ¿Qué grado de compatibilidad hay entre las actuaciones propuestas y dichas tendencias predominantes?

La respuesta a este tipo de cuestiones resultan de gran utilidad para calibrar los condicionantes, las limitaciones y las posibilidades de las diferentes actuaciones a emprender desde el Sector Público, siendo estas precauciones especialmente necesarias cuando, como ocurre actualmente, el nuevo modelo de acumulación contiene un conjunto de elementos que, aislados de su contexto histórico estructural pueden dar pié a consolidar la idea de que todo es posible. A partir de la especialización flexible, la utilización de las nuevas tecnologías, la descentralización, el auge del tejido de pequeñas y medianas empresas y otros ingredientes que se han dado en ciertos distritos industriales, se ha establecido un nuevo paradigma se puede plantear un modelo que en principio se presenta como asequible para aquellos espacios que estén dispuestos a crear la "atmósfera" necesaria como caldo de cultivo para su desarrollo.

Aceptar este planteamiento puede llevar a suponer que un pasado histórico concreto es susceptible de transformarse en cualquier futuro, a pesar de que la experiencia histórica nos ha mostrado hasta qué punto esto no es así, por muy deseable que ese futuro nos parezca. Sin caer en determinismos históricos igualmente inadecuados, no cabe duda de que la percepción de la realidad como proceso nos lleva a la necesidad de considerar la viabilidad, tanto de los objetivos como de las propuestas que se contemplan para alcanzarlos.

De lo contrario, por el camino de aceptar que todo puede conseguirse desde cualquier posición de partida, puede llegarse al convencimiento de que, efectivamente, los actores sociales o institucionales, sin importar las circunstancias en que se encuentren inmersos, tienen todos los grados de libertad para utilizarlos de todos los modos imaginables. Desde esta visión, la planificación puede ser presentada, sobre todo en las sociedades periféricas, en las que el sector privado carece de la potecia necesaria, como la posibilidad para, 
desde el Sector Público, "pilotar" la nave de la economía y la sociedad hacia donde parezca más conveniente y beneficioso para todos.

En el caso de Andalucía, para poder vislumbrar las posibilidades de que la dinámica económica y social pueda orientarse en una u otra dirección, tal vez sea conveniente plantearse ¿Quiénes podrían, aquí y ahora, ser sujetos activos en la búsqueda de nuevos caminos para solucionar los problemas que Andalucía tiene planteados?

En este sentido, la burguesía agraria andaluza, que participó en otros tiempos en la gestión del poder estatal, ejerciéndolo en beneficio propio, tratando de conseguir mejores condicione para la valorización de sus capitales (política de precios agrícolas, planes de regadío, mecanización, crédito agrícola, etc..), pierde su hegemonía a partir de los años 60 , quedando, en gran medida al margen de los procesos de toma de decisiones con el declinar de la agricultura como fuente de riqueza y de poder.

Como es sabido, en ciertas actividades industriales de importancia en Andalucía, como las agroalimentarias, las mejores empresas andaluzas han pasado a engrosar el patrimonio de grandes grupos centrales, mientras que la participación andaluza en la producción agroalimentaria española viene descendiendo, en un proceso de declive sostenido de la industria que parecía tener mayores potencialidades de desarrollo. En general, y a medida que se acentúa la articulación dentro del sistema desde una posición de partida desfavorable, el tejido empresarial andaluz ha experimentado una regresión continua, apareciendo hoy sin fuerzas para impulsar un proyecto de regeneración de la estructura económica de Andalucía desde una perspectiva autóctona.

Por otra parte, en los tiempos que corren, los procesos de crecimiento y acumulación se vertebran alrededor de grandes conglomerados empresariales que condicionan y arrastran, cada vez en mayor medida, las actividades productivas en su conjunto. Las repercusiones de este modelo cobran una enorme importancia en Andalucía, donde la parte medular de los procesos de acumulación y crecimiento tiende a estar radicada, de manera creciente, en las estrategias de esos grandes conglomerados desde los que se modula lo esencial de nuestra dinámica económica.

En este contexto, el empresariado autóctono más próspero se ve impulsado, para poder asegurar la continuidad de su expansión, a diversificar sus 
inversiones, tanto sectorial como territorialmente, imbricándose, cuando no dejándose abserber, en redes y estructuras empresariales que desbordan ampliamente el ámbito andaluz. Se pierden así los límites de su adscripción territorial a la vez que se diluye el papel del empresario individual aislado. Larios. Osborne, Abengoa y algunas otras emblemáticas empresas andaluzas constituyen hoy claros ejemplos de articulación en amplios entramados empresariales que rebasan con creces sus demarcaciones iniciales.

El capital va perdiendo su sentido de pertenencia a un territorio, difuminándose de este modo la posibilidad de identificación del empresariado con un proyecto de transformación social y económica de Andalucía. Con independencia de que, como en otros tiempos, se busquen mecanismos políticos o de otro tipo, que permitan reivindicar y obtener ayudas, subvenciones, mejoras en infraestructuras y otros requisistos para valorizar los capitales.

Se podría pensar en un mayor compromiso con el entorno por parte de pequeños y medianos empresarios, pero en Andalucía las dificultades por las que atraviesa este sector y sus perspectivas ante el realce del papel del mercado y la competitividad como núcleos sobre los que girará la propia superviviencia productiva, los incluye claramente entre los principales damnificados del sistema. Por otra parte, el futuro de estas empresas dependerá en gran medida de hasta qué punto consigan engancharse a circuitos exógenos de crecimiento y acumulación, desde los que termina por imponerse una racionalidad poco compatible con actuaciones filantrópicas hacia el entorno social. De cualquier manera, no parece probable que las actuaciones de este sector alcancen la magnitud necesaria para desencadenar un proceso efectivo de desarrollo autocentrado, cada vez más discordante con las tendencias predominantes en el sistema, que apuntan en dirección contraria.

Ha pasado la hora de esperar que en Andalucía emerja una burguesía autóctona en disposición de conducir a la sociedad y la economía andaluza por caminos que permitan la superación de sus problemas.Hoy, los pueblos periféricos tienen que tomar conciencia de que, para salvar los obstáculos que impiden su desarrollo, están obligados a conducirse a sí mismos.

En estas consideraciones pueden encontrarse algunas de las claves para explicar esa falta de estrategia constatada anteriormente. 
TRABAJO. Revista Andaluza de Relaciones Laborales - n.․․ 2 - Diciembre 1996

\section{Bibliografía}

$-$

BARCELAY, M. (1987): "El socialismo andaluz: la ideología política y la política económica de una comunidad autónoma española" Revista de Estudios Regionales. $\mathrm{n}^{0} 17$.

Barcelay, M. y O’Kean, J.M. (1989): Gestión Pública Estratégica. Conceptos, Análisis y Experiencias. El Caso del IPIA. Instituto de Estudios Fiscales. Consejería de Economía y Fomento (1988): Programa de Desarrollo Económico. 1987-1990.

Consejería de Economía y Hacienda (1991): Plan Andaluz de Desarrollo Económico 1991-1994.

Consejería de Economía, Planificación, Industria y Energía (1985): Plan Económico para Andalucía.

Consejería DE ECONOMIA Y Hacienda (1990): Dos años de Incentivos Regionales en Andalucía.

Delgado Cabeza, M. (1995): "La economía andaluza en los años 90. Rasgos básicos y perspectivas" en Ocho Análisis de la Economía Andaluza. Instituto de Desarrollo Regional. Fundación Universitaria.

Delgado CABEZA, M. y ROMÁN DEl Río, C. (1995): "Impactos territoriales de la reestructuración económica sobre el sector agroalimentario en el sur de Europa.El caso de Andalucía" Revista de Estudios Regionales nº42.

Marchena, M. y Gómez, M.A. (1991): "Ordenación del territorio e incentivos regionales. El caso de Andalucía" Revista de Estudios Regionales $n^{\circ} 30$.

MÁrqUEZ, C. (1993a) "Regional Developement Strategies of a New Regional Governement: The Junta de Andalucía 84-90" Tésis Doctoral inédita.

MÁrquez, C. (1993b): "El Desarrollo de la Red Viaria y Ferroviaria Andaluza en el Período 1987-1992. Impactos económico-territoriales" Revista de Estudios Regionales $n^{\circ} 37$.

Ministerio de ECONomía y Hacienda (1990): Política Regional en 1989.

Morillas RaYA, A. (1995): "Aplicación de la Teoría de Grafos al estudio de los cambios en las relaciones intersectoriales de la economía andaluza en la década de los 80" en Contabilidad Regional y Tablas Input-Output de Andalucía 1990. IEA. 